Instituto Internacional de Investigación y Desarrollo Tecnológico Educativo INDTEC, C.A.

DOI: https://doi.org/10.29394/scientific.issn.2542-2987.2017.2.5.4.66-85

OAI-PMH: http://www.indteca.com/ojs/index.php/Revista Scientific/oai

\title{
Creación de una Unidad de Apoyo Docente que Integran la Tecnología Digital para el Diseño de Medios Didácticos
}

\author{
Autor: Jerry Jesús Peña Morales \\ Universidad Politécnica Territorial de Trujillo "Mario Briceño Iragorry", UPTT "MBI" \\ jerryjpm@gmail.com \\ Trujillo, Venezuela
}

\section{Resumen}

El artículo presenta teoría acerca de la creación de una unidad de apoyo docente integrando la tecnología digital para el diseño de medios didácticos, El objetivo general consiste en generar principios teóricos en la creación de una Unidad de Apoyo Docente que integran la Tecnología Digital para el Diseño de Medios Didácticos. La metodología se sustenta dentro del paradigma positivista, con enfoque cuantitativo, de tipo investigación descriptiva, documental y proyectiva con diseño de campo, considerado un estudio de tipo censal cuya población está constituida por cuarenta y ocho (48) docentes adscritos al Programa Nacional de Formación en Informática, Núcleo Valera de la Universidad Politécnica Territorial del Estado Trujillo Mario Briceño Iragorry. Para la recolección de datos se utilizará la encuesta a través de un cuestionario con dieciocho (18) ítems en escala tipo licker con 5 alternativas de respuestas, cuya validez se realizará mediante el juicio de cinco (5) expertos; mientras que la confiabilidad se determinará mediante el cálculo del coeficiente de Alfa de Cronbach. Entretanto, el aporte significativo es apoyar al profesorado mediante una unidad de apoyo docente para diseñar medios didácticos que sea el protagonista de la innovación pedagógica y andragógicas en los procesos formativos basados en la utilización de las tecnologías digitales.

Palabras clave: tecnología digital; unidades de apoyo docente; medios didácticos. 


\title{
Creation of a Teaching Support Unit that Integrates Digital Technology for the Design of Teaching Media
}

\begin{abstract}
The article presents a theory about the creation of a teaching support unit integrating digital technology for the design of didactic means. The general objective is to generate theoretical principles in the creation of a Teaching Support Unit that integrate Digital Technology for Design of Didactic Means. The methodology is based on the positivist paradigm, with a quantitative approach, of descriptive, documentary and projective research with field design, considered a census type study whose population is constituted by forty eight (48) teachers attached to the National Training Program In Informatics, Nucleus Valera of the Polytechnic University of Trujillo Mario Briceño Iragorry. For data collection, the survey will be used through a questionnaire with eighteen (18) items in licker scale with 5 alternatives of answers, whose validity will be made through the judgment of five (5) experts; While reliability will be determined by calculating the Cronbach's alpha coefficient. Meanwhile, the significant contribution is to support teachers through a teacher support unit to design didactic media that is the protagonist of pedagogical and andragogical innovation in the formative processes based on the use of digital technologies.
\end{abstract}

Keywords: digital technology; teaching support units; didactic means. 


\section{Introducción}

Las Tecnologías de Información y Comunicación (TIC) a los procesos de enseñanza-aprendizaje representa un gran progreso para la creación de una unidad de apoyo docente que integran la tecnología digital para el diseño de medios didácticos. Sin embargo, hay que considerar los costos que deben invertir las universidades en la formación permanente del profesorado, el equipo tecnológico y su posterior mantenimiento. No obstante, el cambio resulta inaplazable y hay que posesionarse del reto, si estamos conscientes de la nueva cultura en la cual estamos sumergidos, donde la universalidad de las TIC es evidente.

De hecho, el crecimiento de los profesores universitarios en relación con sus unidades de apoyo, potenciarían las conductas tales como la iniciativa, curiosidad o la creatividad por la formación, así como una apertura hacia el consumo, uso de la información de sus formas y accesos. Así, se quiere que los docentes universitarios sientan que su aprendizaje y formación sea valiosa para el desarrollo, no sólo su conocimiento práctico sino también metodológico y teórico, en definitiva, que sepa gestionar su tiempo y los espacios de enseñanza-aprendizaje que se propician en la actualidad.

Por esto, el objetivo del presente artículo establece generar principios teóricos en la creación de una Unidad de Apoyo Docente que integran la Tecnología Digital para el Diseño de Medios Didácticos, dado que se observa que no existe una unidad de apoyo docente y poca comprensión sobre las dinámicas de diseño y creación de material didáctico por parte de los profesores universitarios, se traduce en estrategias institucionales poco claras, basadas en propuestas comerciales y con bajo nivel de impacto en el aprendizaje de los alumnos. Ante ello, surge la necesidad de comprender la creación de una unidad de apoyo docente para el diseño de medios de material didáctico en formato digital en los cursos presenciales desde la visión del 
docente universitario a través de los Programas Nacionales de Formación PNF.

En conclusión, se quiere en cuanto a resultados que éstos puedan aportar elementos para estructurar la reflexión de los docentes sobre el tema, al diseño de programas más efectivos de formación docente y de estrategias institucionales de inserción de tecnología más viables y cercanas a los profesores.

\section{Planteamiento del Problema}

Los contextos educativos poseen un enfoque filosófico motivo de su inspiración, es decir son ontologías postmodernas, tal vez atraídas en las obras de Nietzsche desde el pensamiento, el impacto de la tecnología y desde la filosofía de sistemas, un nuevo pensamiento del hombre.

Visto desde esta perspectiva, la complejidad para estudiar la realidad social ha sido considerada como globalización, sobre moderna o postmoderna. Todas estas valoraciones muestran la múltiple interrelación de los aspectos que la conforman y los cambios que desde los distintos niveles de visiones se están operando. A tal efecto Pérez, (1998) establece: "La magnitud de los problemas reclama una nueva sabiduría que permita conciliar el sentido de la existencia, las necesidades de bienestar colectivo, los cambios culturales, los desafíos ecológicos, los conflictos sociales, los impactos de la ciencia y la tecnología, y la formación en valores en el logro de una ciudadanía responsable" (pág. 9).

El fenómeno educativo lleva implícito la transmisión y/o construcción de epistemes ubicados a la capacitación de un recurso humano que sea, entre otros aspectos, crítico, reflexivo, creativo, participativo, innovador, productivo, con valores y alineado con las finalidades educativas.

En este orden de ideas, la Constitución de la República Bolivariana de Venezuela (CRBV: 1999), en los artículos 102 y 103 instituye a los ciudadanos 
venezolanos al disfrute del derecho a acoger una educación de calidad, en correspondencia de oportunidades y condiciones. Asimismo, la Ley Orgánica de Educación (LOE: 2009), garantiza el derecho a una educación de calidad para todos, que sea capaz de asegurar mejoras en las condiciones de vida propias y su comunidad, sin embargo, en la dinámica escolar se observa que las prácticas pedagógicas incompletas traen como consecuencia altos índices de repitencia y deserción escolar, poco rendimiento académico, ausentismo, entre otras.

Esto significa que hay un cambio en la sociedad y cambian las condiciones desde las que emerge el saber.

Esta situación da motivo para el surgimiento de las denominadas Tecnologías de la Información (TIC), tales transformaciones han modificado la sociedad y las condiciones en las que se genera el saber. Ahora ésta forma parte de las universidades, inclusive casi todas en el currículo la han abarcado en sus programas de estudio, el rol del docente, es ser un intermediario en ese proceso del saber y hacer más personalizado, además de ser pedagogo en el uso de las TIC, representante de combinar estas herramientas tecnológicas en la realización de acciones. El impacto en este momento por las nuevas tecnologías en la educación, amerita que los profesores tengan la necesidad de enfrentar retos que implican la entrada de espacios tecnológicos y medios educativos, los cuales provocarán cambios en las universidades y en todo su entorno.

La tecnología digital en el ámbito universitario es un área todavía en Venezuela novedosa dado a que el docente universitario se enfrenta a la decisión de integrar la tecnología en su ejercicio educativo: unos lo hacen y otros no y definitivamente están reacios al cambio.

En ese fin, el profesor universitario lleva al aula su trayectoria académica, humana; que ha recibido en su pasado y aún recibe constantemente. Entretanto, este trabajo tratará sobre la creación de una 
Unidad de Apoyo docente que integran la tecnología digital para el diseño de medios didácticos y que pueden asociarse al papel de las Tecnologías de Información y Comunicación en sus cátedras.

Por esto, se plantearán los aspectos principales de la investigación y para ello se revisará el papel de las TIC en la docencia de acuerdo a la literatura educativa. También se observa el problema del desconocimiento de los significados, acciones y procesos del uso de la tecnología digital en la educación universitaria. Este desconocimiento, impacta en la falta de estructuras para la reflexión personal del docente sobre el uso de las TIC, creyendo conveniente crear la unidad de apoyo docente para el diseño de medios didácticos hacia su formación.

De este modo, las llamadas aplicaciones de uso educativo de la tecnología o software educativos utilizados actualmente en la educación formal son muy variadas. El software educativo digital se diseña y elabora en diversos escenarios: las mismas instituciones educativas con la participación de los maestros y/o entidades de apoyo pedagógico; individuos e instituciones que las hacen disponibles gratuitamente en la red, o empresas comerciales que las ofrecen a la venta.

En este momento, invaden el accionar docente diversos software y contenidos digitales educativos tales como: sistemas administradores de cursos, tutoriales, simulaciones, presentaciones, entre otros, los cuales según De Pablos, (2002), Angulo, (2007) y Díaz Barriga, (2008) "no necesariamente pasan por la consideración pedagógica en su diseño, creación y uso en el proceso de enseñanza-aprendizaje".

Hay muchas universidades que poseen programas de educación a distancia basados en Internet y utilizan diversos apoyos digitales en los cursos de educación presencial. Schmelke y López Ruíz (2003), en la década del 2000 concluyen que: "Hay varias muestras de utilización de las TIC en nuestro 
país, pero poco escrito sobre las experiencias y el impacto de éstas. En general todavía no hay un buen uso de las TIC" (pág. 210).

Concretizando, actualmente se abre un espacio para la reflexión en torno a la Creación de una Unidad de Apoyo Docente que integran la Tecnología Digital para el Diseño de Medios Didácticos situación evidente en el análisis de las prácticas pedagógicas centradas en contenidos y no en procesos. Al respecto, se propone considerar al educador, como punto de partida de un proceso, que permita pasar del énfasis de las gruesas informaciones descontextualizadas a la dinámica del diálogo de saberes, y también a un acercamiento de los estudiantes al conocimiento de las Tecnologías de Información y Comunicación, ligadas con la didáctica del docente, con la forma como asume su quehacer y cómo él se relaciona con la tecnología.

Visto de esta forma este trabajo busca orientar, respecto a la creación de una unidad de apoyo docente para el diseño de medios didácticos en la Universidad Politécnica Territorial del Estado Trujillo, ubicada en parroquia Juan Ignacio Montilla, municipio Valera, estado Trujillo. Por tanto, se formula la siguiente interrogante. ¿Cuáles aspectos de las unidades de apoyo docente inciden en el diseño de medios didácticos durante el proceso de la enseñanzaaprendizaje basada en la tecnología de la información y comunicación?

\section{Bases Teóricas}

El marco teórico, concibe el relato al basamento figurado que sustenta esta investigación, la cual está relacionada con la creación de una Unidad de Apoyo Docente que integra la Tecnología Digital para el Diseño de Medios Didácticos. Por esto, se plantean las figuras teóricas cuya intencionalidad medular consiste en hacer una sistematización de aquellas teorías antecedentes, que ayudarán en el problema a investigar y por ende, pueden 
servir a otros estudios que sin duda, contribuirán con conocimientos novedosos al estudio.

\subsection{Perfil del Docente Universitario}

Conjunto de capacidades que asemejan la formación del sujeto, para asumir las responsabilidades propias del desarrollo de funciones y tareas de una profesión. En el caso del perfil del docente universitario, definir un perfil apropiado, adecuado a la multiplicidad y a los cambios que se vienen dando en la sociedad actual.

El perfil competitivo es asociado a una imagen de docencia anhelada y que se compone en la responsabilidad de tomar decisiones de políticas educativas. Así, su rol vendrá encuadrado en un modelo sistémico e interdisciplinar, donde la docencia, investigación, su saber, saber hacer y querer hacer conformará su accionar educativo.

Estas transformaciones pedagógicas formulan mejoras de la calidad y adquisición del conocimiento, más en los actuales momentos son determinadas por la incorporación de las TIC, como un elemento significativo para conseguir innovaciones en las instituciones educativas en los diferentes ambientes de aprendizaje. De igual manera, el Diseño Curricular del Sistema Educativo Bolivariano (2007), en los fines y principios establece que "se debe promover el empleo de las TIC, desde un enfoque social y la formación en, por y para el trabajo productivo y liberador, que contribuya con el adecuado uso de la ciencia y la tecnología" (pág. 22-23).

Es decir, el estudiante es el centro del accionar docente: la valoración de su trabajo, horas de estudio, prácticas, trabajos en grupo, tutorías, serán el eje de acción con éstos, dando una nueva función a la figura del profesor, pasando a ser un guía, orientador en la información relevante y en la búsqueda de bibliografías relacionadas con el estudio pertinente. Así, todo lo expuesto, acerca del perfil del docente se hizo con la intención de ahondar en 
fundamentos filosóficos, es decir, significa rescatar los elementos que permiten entender el ser y el estar del sujeto social en la realidad, entendiendo este quehacer ideológico que en la tradición teórica clásica es llamada "ontología" aunado a principios éticos que sustentan la acción educativa.

\subsection{Episteme de la Sociedad del Conocimiento}

La óptica en Latinoamérica se encuentra en plena búsqueda de caminos propios y fragmentos hacia el desarrollo, impuestos por intereses que desvían a los pueblos de los propios. Existen diversos niveles de noción, conceptos y categorías en un mismo fenómeno y que la posibilidad de avanzar en la creación de saberes en esta época de ruptura de paradigmas se encuentra en aceptar el riesgo de hacerlo, es decir, aceptando que nuestro abordaje puede llegar a conclusiones totalmente distintas de otro y que en esa diversidad es donde ahora se pudiera encontrar el estatuto de la verdad, lo que sería totalmente contrario a la esencia ortodoxa de dicha categoría filosófica.

Se infiere entonces, que el pueblo al participar en la sociedad del conocimiento lo hará desde otro lugar y con un conocimiento alternativo, a juicio de Córdoba, (1995). "...se trata de ubicarse en la dialéctica rico-pobre, sino en la otredad, y esto viene dado por la comunicación y el diálogo, que, a su vez, implica la ruptura epistémica de todo un mundo de vida" (Pag. 84). En otras palabras, los cambios ocurridos en las distintas sociedades a lo largo de la existencia del hombre han sido tan abundantes como los intentos del individuo en aprovechar el medio donde está inmerso para su propio beneficio.

Es decir que la información no está relegada a ser transmitida de sujeto a sujeto, por medio del lenguaje oral como era en las primeras apariciones históricas, sino que ahora tenemos nuevas formas que llegan de la mano de las tecnologías empleadas, que admiten comunicarnos mediante un chat $o$ 
video conferencia con otra persona o personas en otra parte del planeta gracias a los avances tecnológicos que se están dando actualmente.

Web se aplican como una nueva filosofía, que según Casamayor, (2008) "sitúa al participante en el centro del proceso de aprendizaje, el cual es construido 
en colaboración con sus pares, los profesores y el contexto entero a su alrededor" (pág. 91). Es por ello, se puede decir que es una nueva etapa de la Internet, nombrada desde el 2004 como la Web 2.0, compuesta en una compacta plataforma tecnológica como efecto de la interacción, colaboración y retroalimentación de los propios usuarios. Sin duda, las herramientas Web no se produjeron con el propósito de educar, muchas de estas aplicaciones se han enfocado hacia diversos argumentos sociales y educativos, incluyendo el ámbito universitario.

\subsubsection{El Aprendizaje Colaborativo}

Resalta sus efectos sobre la dinámica del grupo, la integración y motivación de los estudiantes, así como los propios resultados del aprendizaje.

Presta menos atención a los casos en los que no funciona como se espera, según Rodríguez, (2001) "cuando se producen varios efectos no deseados referidos a la no colaboración, el que miembros de un equipo utilicen el trabajo del resto, o simplemente el que un individuo se aproveche de los resultados para fines propios, aunque en general se consideren menores que sus ventajas" (pág. 91).

Se trata de un campo práctico que combina una tradición pedagógica, con un conjunto de saberes sobre las formas organizativas de las acciones educativas y sobre el que hay relativamente pocos conocimientos psicológicos y pedagógicos.

\section{Metodología}

La naturaleza de este estudio consta del abordaje tanto del tipo como del diseño de la investigación los cuales definen en primer término el alcance de la misma, así como la estrategia adoptada para responder a la problemática existente, tal como lo sugiere Arias (2016: 27). Aunado a ello, la claridad con la cual se exprese esta naturaleza conlleva a que el investigador tenga 
claramente establecido lo que va a hacer y al mismo tiempo cómo lo va a realizar.

En ese orden de ideas, el tipo de investigación asumido en esta oportunidad es proyectiva, la cual según Hurtado (2012: 122) consiste en una propuesta de alternativas de cambio, sobre la base de un proceso previo de indagación.

Es de hacer notar que, en esta investigación el diagnóstico se realiza cuando se generar teorías para la creación de una unidad de apoyo docente que integran la tecnología digital para el diseño de medos didácticos, de acuerdo a las cuales se establecerán los aportes teóricos que fundamentan la misma en la Universidad Politécnica Territorial del Estado Trujillo Núcleo Valera. Por lo que estos insumos explicarán el comportamiento de las mismas en la realidad del contexto.

\subsection{Postura Paradigmática}

Desde el punto de vista epistemológico, el estudio denominado creación de una Unidad de Apoyo Docente que integran la Tecnología Digital para el Diseño de Medios Didácticos, se enmarcará dentro del paradigma positivista, con enfoque cuantitativo, de tipo investigación descriptiva y documental. Al respecto, Padrón, (2004)

"en el paradigma positivista los sucesos del mundo tanto materiales como humanos, por más disímiles e inconexos que parezcan, obedecen a ciertos patrones cuya regularidad puede ser establecida gracias a la observación de sus repeticiones" (pág. 110).

\subsection{Enfoque Cuantitativo}

El paradigma con enfoque cuantitativo, se fundamenta en el positivismo, el cual percibe la igualdad de los fenómenos, emplea la idea hipotéticadeductiva como explicación y enseña que la materialización del dato, es la 
consecuencia de procesos derivados de la experiencia. Esta noción se organiza sobre la base de términos de operacionalización que admiten desarreglar el todo en sus partes y completar éstas para lograr el todo. Al respecto, Comte, 1978 citado en Palella y Martins (2010) establece "el espíritu humano debe renunciar a conocer el ser mismo (la naturaleza) de las cosas y contentarse con las verdades que proporcionan la observación y la experimentación" (pág. 40).

\subsection{Técnica e Instrumento}

Como técnica de investigación se manejará la encuesta, la cual según Kerlinger y Lee, (2003) permite estudiar;

"poblaciones (o universos) grandes, pequeñas seleccionando y analizando muestras elegidas de la población para descubrir la incidencia relativa, distribución e interrelaciones de las variables sociológicas y psicológicas" (pág. 427).

El instrumento que se utilizará es el cuestionario, el cual según Hernández, Fernández y Baptista, (2006) consiste en "un conjunto de preguntas respecto de una o más variables a medir". Se elaborará dos cuestionarios, uno para los docentes y otro para los estudiantes.

\section{Análisis de Resultados}

Este apartado recopila la información obtenida luego de la recolección y procesamiento de los datos, mostrando los resultados obtenidos en el cumplimiento de cada uno de los objetivos específicos. El investigador analiza el comportamiento de los docentes con respecto a la integración de las unidades curriculares y si utilizan la tecnología para el diseño de medios didácticos.

En ese orden de ideas, cada cuadro contiene la información de los indicadores que conforman la dimensión sujeta a medición para dar respuesta 
al objetivo específico correspondiente, para el cual se establece la contrastación de los resultados con la delimitación teórica establecida como fuente esencial para determinar los elementos fundamentales que son utilizados de insumos para la elaboración de la teoría planteada como objetivo general de esta investigación.

Cuadro 1. Habilidad docente en Integración de las unidades curriculares.

\begin{tabular}{|c|c|c|c|c|c|c|}
\hline \multirow{2}{*}{\begin{tabular}{|l} 
Indicadores \\
$\begin{array}{c}\text { Alternativas de } \\
\text { respuesta }\end{array}$
\end{tabular}} & \multicolumn{2}{|c|}{$\begin{array}{c}\text { Vinculación tipo } \\
\text { de uso de la } \\
\text { tecnología - }\end{array}$} & \multicolumn{2}{|c|}{$\begin{array}{l}\text { Estimulación del } \\
\text { conocimiento } \\
\text { pertinente }\end{array}$} & \multicolumn{2}{|c|}{$\begin{array}{l}\text { Transferencia de } \\
\text { los contenidos }\end{array}$} \\
\hline & FA & FR & FA & FR & FA & FR \\
\hline Siempre & 5 & 10,42 & 12 & 25,00 & 14 & 29,17 \\
\hline Casi Siempre & 11 & 22,92 & 12 & 25,00 & 17 & 35,42 \\
\hline Algunas Veces & 10 & 20,83 & 10 & 20,83 & 8 & 16,67 \\
\hline Casi Nunca & 13 & 27,08 & 9 & 18,75 & 6 & 12,50 \\
\hline Nunca & 9 & 18,75 & 5 & 10,42 & 3 & 6,25 \\
\hline Total, respuestas & 48 & 100 & 48 & 100 & 48 & 100 \\
\hline \multicolumn{7}{|c|}{ Estadísticos por indicador } \\
\hline Media & \multicolumn{2}{|c|}{2,79} & \multicolumn{2}{|c|}{3,35} & \multicolumn{2}{|c|}{3,65} \\
\hline Categoría & \multicolumn{2}{|c|}{$\begin{array}{l}\text { Moderada } \\
\text { presencia }\end{array}$} & \multicolumn{2}{|c|}{ Moderada presencia } & \multicolumn{2}{|c|}{ Alta presencia } \\
\hline $\begin{array}{l}\text { Desviación } \\
\text { estándar }\end{array}$ & \multicolumn{2}{|c|}{1,25} & \multicolumn{2}{|c|}{1,33} & \multicolumn{2}{|c|}{1,24} \\
\hline Categoría & \multicolumn{2}{|c|}{ Baja dispersión } & \multicolumn{2}{|c|}{ Baja dispersión } & \multicolumn{2}{|c|}{ Baja dispersión } \\
\hline
\end{tabular}

Fuente: Cálculos basados en las respuestas del cuestionario aplicado a docentes del Programa Nacional de Formación en Informática (PNFI) Extensión Trujillo de la UPTTMBI. Peña (2017).

Al analizar el indicador vinculación tipo de uso de la tecnología resultados del aprendizaje, el cuadro 1 muestra que la tendencia de imparcial a negativa con respecto al mismo se acerca al $70 \%$ de las opiniones de los encuestados, teniendo en contraposición a un poco más del $30 \%$ quienes opinan en que siempre o casi siempre llevan a cabo la referida vinculación. 
Con respecto a la habilidad para la estimulación del conocimiento pertinente, se tiene una postura dividida entre quienes opinan positivamente y quienes asumen una tendencia de imparcial a negativa, evidenciando un 50\% para ambas posturas al aprender-haciendo con las TIC

Como siguiente indicador mostrado en el cuadro anterior, se tiene la transferencia de los contenidos cuya postura a favor por parte de los docentes encuestados supera la barrera del $60 \%$ de las opiniones, no obstante, es indispensable denotar la existencia de un $16,67 \%$ de respuestas que muestran cierta indiferencia al respecto, aunado a un poco menos del $20 \%$ de las respuestas con tendencia a desfavorecer el mencionado indicador.

En ese orden de ideas, al visualizar de manera conjunta la habilidad docente en Integración de las unidades curriculares, el cuadro 1 muestra una moderada presencia de estas habilidades en los docentes encuestados. Ahora bien, al disgregar los resultados, se tiene que la transferencia de contenidos es la habilidad con mayor aceptación por parte de los docentes, seguido por la estimulación del conocimiento pertinente y por último, de forma contradictoria la vinculación entre el tipo de uso de la tecnología y los resultados del aprendizaje.

Esta última afirmación pareciera ser incoherente con la población objeto de estudio, dado que los docentes pertenecen al Programa Nacional de Formación en informática donde la tecnología es la herramienta fundamental en el desarrollo de las actividades académicas. De allí que, los resultados obtenidos, donde deben incorporarse las tecnologías digitales no se está haciendo realmente y se debe buscar una respuesta para mejorar la incorporación de la tecnología en las unidades curriculares de los docentes.

Ahora bien, en el constructo teórico se plantea sine qua non la presencia del componente axiológico como ente regulador de los comportamientos generados con la creación de la unidad de apoyo docente. 
En ese sentido, se plantean tres (3) conceptos base como son: competencias, orientaciones tecnológicas e instrumentalidad tecnológica y dentro de los supuestos axiológicos emergentes se tiene:

a). Responsabilidad, requerida sustancialmente cuando se habla de tecnología pues el alcance de estas puede afectar el contexto del usuario y traspasar las fronteras físicas existentes. La responsabilidad es considerada como la capacidad de asumir las consecuencias de los actos realizados y responder ante las mismas.

b). Ética, es evidente que la tecnología trae consigo el manejo de volúmenes de información considerables. Es así como la ética, es entendida como el comportamiento ajustado a normas preestablecidas en común acuerdo dentro de la comunidad académica que garantice la armonía en los espacios universitarios.

c). Respeto, término referido al comportamiento asumido por los usuarios de las TIC en función de establecer posiciones personales, académicas y gerenciales sin afectar negativamente los entornos de aprendizaje ni a los actores que forman parte de ellos; reconociendo la presencia de otros con los mismos derechos y deberes.

d). Compromiso, está relacionado a la capacidad del individuo de asumir como parte de su propia naturaleza determinadas acciones que van a favor de obtener resultados ajustados a metas preestablecidas. Para el caso de la unidad de apoyo docente, el compromiso representa la oportunidad de internalizar la aplicabilidad de la tecnología como una herramienta para el desarrollo y mejoramiento de las unidades curriculares con el diseño de medios didácticos.

\section{Conclusiones}

La Unidad de Apoyo Docente representa la oportunidad tener un lugar en el ámbito universitario para el uso y aplicación de las mismas para diseñar 
a través de los medios didácticos las unidades curriculares y desarrollar estrategias pedagógicas para cumplir con los objetivos establecidos en el currículo. En ese sentido, al reconocer la creciente innovación en materia tecnológica, aunado a los acelerados cambios propios de una sociedad característica del siglo XXI; demanda del contexto universitario el impulso y la consolidación de profesionales emprendedores e innovadores que le den a la tecnología el uso adecuado y al mismo tiempo, internalicen su aplicabilidad para el beneficio del colectivo.

En cuanto a la habilidad docente en Integración de las unidades curriculares, los resultados muestran una moderada presencia de estas en los docentes encuestados, encontrando un repunte en la transferencia de contenidos y la estimulación del conocimiento pertinente, por encima de la vinculación entre el tipo de uso de la tecnología y los resultados del aprendizaje. Vale acotar que, este último resultado parece inconsistente ante un contexto relacionado con la informática; sin embargo, la falta de seguimiento de estas habilidades deja abierta la necesidad de establecer orientaciones tecnológicas que fortalezcan el uso de las Tecnologías de Información y Comunicación.

Ahora bien, al establecer los aportes teóricos que fundamentan la creación de una unidad de apoyo docente utilizando la tecnología digital a través de los medios didácticos resultó innovador la generación de los mismos partiendo de las debilidades y requerimientos hallados en la medición de la variable, por lo que se estableció un nuevo dimensionamiento donde se consideran las competencias, orientaciones tecnológicas e instrumentalidad tecnológica como constructos que permitieron definir la apropiación de las TIC, enfatizando la presencia de una base axiológica. Ante estos resultados, se tiene el punto de partida para el desarrollo de la propuesta donde se redefine la variable como esencia misma de la teoría planteada. 


\section{Consideraciones Finales}

El proyecto de creación de una unidad de apoyo docente con tecnología digital para diseñar medios didácticos apuesta por un modelo en el que el profesorado es el protagonista de la innovación pedagógica. De este modo, el material se aproxima en un pretexto o estrategia para la mejora y desarrollo profesional de los docentes, para acercar personas adultas a los procesos formativos basados en la utilización de las tecnologías digitales.

La creación de una unidad de apoyo docente facilita la innovación pedagógica e integración de las nuevas tecnologías en las prácticas docentes en todos los niveles.

\section{Referencias}

Arias, F. (2016). El proyecto de la investigación. (7a ed.). Caracas: Epísteme.

Córdova, V. (1995). Hacia una Sociología de lo vivido. Fondo Editorial Tropykos-FACES-UCV. Caracas.

Hurtado, J. (2012). El proyecto de investigación. Comprensión holística de la metodología y la investigación. (7ma ed.). Caracas: Quirón. ISBN No 978-95844-3440-1

Ley Orgánica de Educación (LOE: 2009). Gaceta Oficial No 5.929 Extraordinaria, del 15 de Agosto de 2009, Caracas.

Nietzsche, F. (1835). Así habló Zaratustra. Edición de Andrés Sánchez Pascual en "El libro de bolsillo", "Biblioteca de autor". Alianza Editorial, 2006. Págs. 36:37.

Pérez, Limardo, J. (1998). Problemas Existenciales para el Cambio. Colombia.

Rodríguez, J. (2011). Discursos, Poder y Saber en la Formación Permanente: La Perspectiva del Profesorado sobre la Integración Curricular de las TIC. Tesis Doctoral en Universidad de Alcalá para 
optar al Título de Dr. en Ciencias de la Educación. Departamento de Didáctica.

Ministerio del Poder Popular Para la Educación (2007). Diseño Curricular del

Sistema a la vez Bolivariano. CENAMEC. Caracas, Venezuela.

Hernández, Fernández y Baptista R. (2006). Metodología de la Investigación. México: Editorial McGraw-Hill.

Casamayor, G. (2008). La Formación on-Line. Barcelona. Grao.

De Pablos y otros (2008). EI Espacio Europeo de Educación Superior y las

Tecnologías de la Información y la Comunicación. Percepciones y

Demandas del Profesorado. Revista de Educación (337), 99-124.

Kerlinger y Lee (2003). Investigación y Comportamiento. México Mac Graw. Palella, S. y Martins, F. (2010). Metodología de la Investigación Cuantitativa. Tercera edición. Caracas: Fondo Editorial de la Universidad Pedagógica Libertador (FEDUPEL).

Padrón, J. (2004). Conferencia Inaugural en las X Jornadas de Investigación: Investigación y Transformación Social. Caracas, Venezuela. Marzo. [Documento en línea]. Recuperado de: http://padron.entretemas.com.ve/Inv TransfSoc/index.htm 


\section{Jerry Jesús Peña Morales}

e-mail: jerryjpm@gmail.com

Nacido en Caracas, Venezuela. Graduado en Ingeniería de Sistemas, cursado en Universidad de Los Andes. Actualmente curso estudios de Doctorado en Ciencias de la Educación en Universidad Nacional Experimental "Rafael María Baralt". Magister en Ingeniería de Control de Procesos en Universidad Experimental Politécnica. Barquisimeto. Experiencia Laboral: Actualmente en Instituto Universitario de Tecnología del Estado Trujillo. Cargo: Docente Contratado (Tiempo Completo) Desde Abril 1998. Asesor de Sistemas de Información y programación. Además, puedo capacitar a cualquier personal en el área de ofimática (procesador de texto, hoja de cálculo, presentaciones) y en el área de informática en el sistema operativo Windows y Linux. 